\title{
Oriented orbifold vertex groups and cobordism and an associated differential graded algebra
}

\author{
KIMBERLY DRUSCHEL
}

\begin{abstract}
We develop a homology of vertex groups as a tool for studying orbifolds and orbifold cobordism and its torsion. To a pair $(G, H)$ of conjugacy classes of degree- $n$ and degree- $(n-1)$ finite subgroups of $\mathrm{SO}(n)$ and $\mathrm{SO}(n-1)$ we associate the parity with which $H$ occurs up to $O(n)$ conjugacy as a vertex group in the orbifold $S^{n-1} / G$. This extends to a map $d_{n}: \beta_{n} \rightarrow \beta_{n-1}$ between the $Z_{2}$ vector spaces whose bases are all such conjugacy classes in $\mathrm{SO}(n)$ and then $\mathrm{SO}(n-1)$. Using orbifold graphs, we prove $d: \beta \rightarrow \beta$ is a differential and defines a homology, $\mathcal{H}_{*}$. We develop a map $s: \beta_{*}^{-} \rightarrow \beta_{*+1}^{-}$for a subcomplex of groups which admit orientation-reversing automorphisms. We then look at examples and algebraic properties of $d$ and $s$, including that $d$ is a derivation. We prove that the natural map $\psi$ between the set of diffeomorphism classes of closed, locally oriented $n$-orbifolds and $\beta_{n}$ maps into $\operatorname{ker} d_{n}$ and that this map is onto $\operatorname{ker} d_{n}$ for $n \leq 4$. We relate $d$ to orbifold cobordism and surgery and show that $\psi$ quotients to a map between oriented orbifold cobordism and $\mathcal{H}_{*}$.
\end{abstract}

57R18, 57R90; 55N32, 57R65

\section{Introduction}

The extension of manifold cobordism to orbifold cobordism began with the study of rational oriented orbifold cobordism by the author [4], where invariants and generators were determined. However the actual oriented orbifold cobordism ring and its torsion remain open problems. For example, it is not known whether the odd-dimensional oriented orbifold cobordism groups are trivial, even though they are trivial rationally. In [5] we presented machinery for controlling the local groups and singularities. In addition, in that paper we used that machinery to show that all oriented orbifolds in dimensions less than or equal to three bound orientably. Two main steps in the proofs for those dimensions involve the folding of some vertex groups to create a cobordism to an orbifold with simpler vertex groups and noting that only certain combinations of vertex groups can occur in a compact, oriented, 3-dimensional orbifold. When $G$ is a finite degree- $n$ subgroup of $\mathrm{SO}(n)$, some multiple of the sphere quotient orbifold $S^{n-1} / G$ 
orientably bounds an orbifold with set of local groups no more than those found in $S^{n-1} / G$ (see Proposition 3.8). Hence one can construct a closed oriented $n-$ dimensional orbifold whose only vertex groups are $G$. However the minimum number of points labelled by $G$ is not necessarily known. In this paper we begin to address the question of which precise combinations of finite degree- $n$ linear groups, including repetitions, can occur in an oriented or locally oriented $n$-dimensional orbifold by providing a first obstruction for this.

Thus to each finite degree- $n$ group we associate the parity of the labelled points for each vertex group type on its sphere quotient. We extend this to linear combinations of such linear groups and so build a differential $d$ associated with finite degree- $n$ subgroups of $\mathrm{SO}(n)$, with $n$ varying, and hence obtain a homology. This homology is trivial for dimensions up through three but nontrivial in dimension four. If a locally oriented orbifold has vertex groups $G_{1}, G_{2}, \ldots, G_{k}, d$ applied to the sum of these must be zero. Hence we have a map from the set of diffeomorphism classes of locally oriented orbifolds to ker $d$. In addition this map quotients to one from the oriented orbifold cobordism ring to the homology of $d$ (see Theorem 3.12).

In degrees two through four, if $d Z=0$ for some linear combination $Z$ of degree $n$ linear groups, then there is an oriented $n$-orbifold with those precise vertex groups (see Theorem 3.13). As another example, when $G$ has no direct summands of real type other than $\left\langle-\mathrm{id}_{2 n}\right\rangle$, then $d$ is zero (see Proposition 2.2), and one can also construct an oriented orbifold whose only singular vertex has local group $G$ (see Proposition 3.9).

We calculate examples of $d$ and also provide formulas for $d$ for direct sums and direct products (see Proposition 2.1 and Corollary 2.3). The direct product formula shows us that $d$ is in fact a derivation. In the case where $G$ admits an orientation-reversing linear automorphism $u$ with $u^{2} \in G$, we construct a semidirect product of $G$ by $(u,-1)$ in degree $n+1$. We present equations for calculating $d$ of this semidirect product and relate it to $d G$ (see Theorem 2.12). One important tool we use is the orbifold graph of this semidirect product. These semidirect products afford us a map $s: \beta_{*}^{-} \rightarrow \beta_{*+1}^{-}$for the subcomplex built from vertex groups which admit such an automorphism. With this and a further decomposition of $\beta_{n}^{-}$we make some headway in calculating the homology $\mathcal{H}_{*}$.

The last section is where we concentrate on the orbifold definitions, including surgery on an orbifold, and discuss how $d$ and the homology relate to orbifolds and their cobordism and surgery.

The author would like to thank the referee for helpful comments and suggestions. 


\section{The differential $d$ and its homology}

For the subgroups $A, A^{\prime}$ of $B$, let $A \sim_{B} A^{\prime}$ indicate that $A$ and $A^{\prime}$ are conjugate in $B$ and $(A)_{B}$ be the conjugacy class of $A$ in $B$. The normalizer and then centralizer of $A$ in $B$ are denoted by $N_{B}(A)$ and $C_{B}(A)$. If we omit $B$ we are implying, unless otherwise stated, that it is $O(n)$, the orthogonal group, for some appropriate $n$.

The antipodal element of $O(n)$ is written as $-\mathrm{id}_{n}$.

For $g \in O(n)$ and $b= \pm 1,(g, b)$ provides inclusions of $O(n) \times O(1)$ and $O(n)$ in $O(n+1)$. For an integer $n,[n]_{2}$ will indicate the parity of $n$ or $n \bmod 2$.

Definition 1.1 A subgroup $G<O(n)$ is of degree $n$ if it has no trivial direct summand. For $G$ and $H$ finite, degree- $n$, respectively degree- $(n-1)$, subgroups of $O(n)$, respectively $O(n-1)$, we say $H$ is a vertex group for $G$ if there is an isolated singular point in the sphere quotient orbifold $S^{n-1} / G$ whose local group is conjugate to $H$ in $O(n)$, or equivalently, there is a line in $\mathbb{R}^{n}$ whose $G$-isotropy group is conjugate to $H$ in $O(n)$. We would also say $H$ is a vertex group for the orbifold $S^{n-1} / G$. We denote the $O(n)$ conjugacy classes of vertex groups of an orbifold $Q$ by $\mathcal{G}_{Q}^{v}$ and we abbreviate $\mathcal{G}_{S^{n-1 / G}}^{v}$ by $\mathcal{G}^{v}(G)$.

Definition 1.2 Let $\mathcal{L}(G)$ represent the set of $G$ conjugacy classes of subgroups of $G$ which are vertex groups of $G$. For $(H) \in \mathcal{G}^{v}(G), \mathcal{L}(G, H)$ will be those $(K)_{G} \in \mathcal{L}(G)$ which are $O(n)$ conjugate to $H$.

Remark $1.3(K)_{G} \in \mathcal{L}(G)$ if and only if $K$ is a maximal degree-(n-1) subgroup of $G$.

Suppose $(K)_{G} \in \mathcal{L}(G, H)$ and $K$ is the isotropy group of $\ell$, a line in $\mathbb{R}^{n}$. Since $N_{G}(K)$ sends $\ell$ to itself, it is either $K$ or is degree- $n$ and a $Z_{2}$-extension of $K$ with the $Z_{2}$ element folding $\ell$. Accordingly the $G$ conjugacy class of $K$ contributes two or one singular points in $S^{n-1} / G$ with vertex group $H$.

Definition 1.4 Let $\mathcal{L}(G)_{-}$and $\mathcal{L}(G, H)_{-}$denote those classes $(K)_{G}$ in $\mathcal{L}(G)$ and $\mathcal{L}(G, H)$ for which $N_{G}(K) / K=Z_{2}$. In addition, $\mathcal{L}(G)_{+}$represents $\mathcal{L}(G)-\mathcal{L}(G)_{-}$ and similarly for $\mathcal{L}(G, H)_{+}$. For $(K)_{G} \in \mathcal{L}(G)$ let $p_{K, G}=\left[\left|N_{G}(K) / K\right|\right]_{2}$ and $n_{K, G}=\left[1+p_{K, G}\right]_{2}$. Then $n_{K, G}$ is the parity of the singular points with vertex group $H$ in $S^{n-1} / G$ contributed by $K$.

Definition 1.5 Let $\mathcal{S O}_{n, f}$ be the set of $O(n)$ conjugacy classes of finite degree- $n$ subgroups of $\mathrm{SO}(n)$.

Let $\lambda_{H}(G) \in Z_{2}$ be the parity of singular points in $S^{n-1} / G$ with vertex group $H$. 
Note that $\lambda_{H}(G)$ is independent of the representatives $G$ for $(G)$ and $H$ for $(H)$ and so defines a map from $\mathcal{S O}_{n, f} \times \mathcal{S O}_{n-1, f}$ to $Z_{2}$.

Example 1.6 For $\mathcal{I}$ the icosahedral group of degree 3, $\lambda_{\mathcal{C}_{n}}(\mathcal{I})=1$ for $n=2,3,5$, and is 0 otherwise. Here $\mathcal{C}_{n}$ represents the cyclic subgroup of $\mathrm{SO}(2)$ of order $n$.

Proposition 1.7

(i) $\lambda_{H}(G)=\left[\sum_{(K)_{G} \in \mathcal{L}(G, H)} n_{K, G}\right]_{2}$.

(ii) $\lambda_{H}(G)=\left[\left|\mathcal{L}(G, H)_{-}\right|\right]_{2}$.

Proposition 1.8 In the following two cases, $\mathcal{L}(G, H)=\mathcal{L}(G, H)_{-}$, and so $\lambda_{H}(G)=$ $[|\mathcal{L}(G, H)|]_{2}$ for all $(H)_{G} \in \mathcal{L}(G)$ :

(i) $G$ contains $-\mathrm{id}_{n}$.

(ii) $G$ is nilpotent.

Proof For (i) every line is then folded. For (ii) a nilpotent group has the property that every proper subgroup is a proper subgroup of its normalizer (see Isaacs [7, Theorem 1.22]) and so $N_{G}(K) / K=Z_{2}$. Also, nilpotent linear groups are monomial (see Dixon [3, Problem 10.14]).

Definition 1.9 Denote by $\beta_{n}$ the $Z_{2}$ vector space indexed by $\mathcal{S O}_{n, f}$ and by $[G]$ the basis element of $\beta_{n}$ indexed by the conjugacy class $(G) \in \mathcal{S O}_{n, f}$, or the subspace spanned by it.

Definition 1.10 Define $d_{n}: \beta_{n} \rightarrow \beta_{n-1}$ by defining it on the basis element $[G]$ as $d_{n}[G]=\sum_{\mathcal{S O}_{n-1, f}} \lambda_{H}(G)[H]$, and then extending it linearly. Let $\beta=\bigoplus_{n \in \mathbb{N}} \beta_{n}$ and $d: \beta \rightarrow \beta$ be defined on $\beta_{n}$ as $d_{n}$.

We have $\beta_{1}=\beta_{0}=0$ and $\beta_{2}=\bigoplus_{k \geq 2}\left[\mathcal{C}_{k}\right]$, where $\mathcal{C}_{k}$ is the cyclic subgroup of order $k$ of $S^{1}=\mathrm{SO}(2)$. In addition, $d_{2}=0$.

Proposition 1.11 We have $\beta_{3}=[\mathcal{I}] \oplus[\mathcal{O}] \oplus[\mathcal{T}] \bigoplus_{k \geq 2}\left[\mathcal{D}_{k}\right]$. Here $\mathcal{I}, \mathcal{O}, \mathcal{T}, \mathcal{D}_{k}$ are the icosahedral, octahedral, tetrahedral and the dihedral groups of order $2 k$. We have the following:

$$
\begin{aligned}
d[\mathcal{I}] & =\left[\mathcal{C}_{5}\right]+\left[\mathcal{C}_{3}\right]+\left[\mathcal{C}_{2}\right] . \\
d[\mathcal{O}] & =\left[\mathcal{C}_{4}\right]+\left[\mathcal{C}_{3}\right]+\left[\mathcal{C}_{2}\right] . \\
d[\mathcal{T}] & =\left[\mathcal{C}_{2}\right] . \\
d\left[\mathcal{D}_{n}\right] & =\left[\mathcal{C}_{n}\right] .
\end{aligned}
$$


Proof The finite subgroups of $\mathrm{SO}(3)$, up to conjugacy, are those listed above; see Du Val [6, Section 2.7]. The orbifolds $S^{2} / G, G$ as above, have three singular points which have local groups $\mathcal{C}_{k_{i}}, i=1,2,3$. The values of $\left\{k_{1}, k_{2}, k_{3}\right\}$ are $\{2,3,5\}$ for $\mathcal{I}$, $\{2,3,4\}$ for $\mathcal{O},\{2,3,3\}$ for $\mathcal{T}$ and $\{2,2, n\}$ for $\mathcal{D}_{n}$.

Example 1.12 Consider the family of linear groups $s^{k} \mathcal{C}_{2}<\mathrm{SO}(k+2), k \geq 0$, defined by $s^{0} \mathcal{C}_{2}=\mathcal{C}_{2}$ and $s^{k+1} \mathcal{C}_{2}=\left\langle s^{k} \mathcal{C}_{2}, g\right\rangle$, where $g$ is the diagonal element of $\mathrm{SO}(k+3)$ with diagonal $(1, \ldots, 1,-1,-1)$. We have that $s^{1} \mathcal{C}_{2}=\mathcal{D}_{2}$ and the singular set in $S^{2} / s^{1} \mathcal{C}_{2}$ is three points labelled by $\mathcal{C}_{2}$. For $k>1$ the singular set of dimensions 0 and 1 in $S^{k+1} / s^{k} \mathcal{C}_{2}$ is the complete graph $K_{k+2}$ with vertices labelled $s^{k-1} \mathcal{C}_{2}$ and edges labelled $s^{k-2} \mathcal{C}_{2}$. We illustrate this for $s^{2} \mathcal{C}_{2}$ and $s^{3} \mathcal{C}_{2}$ in Figure 1 below. The picture of the graph on the sphere quotient surrounding a labelled vertex in Figure 1(b) would be Figure 1(a).

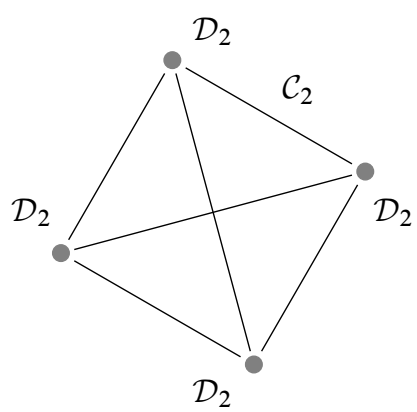

(a) $s^{2} \mathcal{C}_{2}$

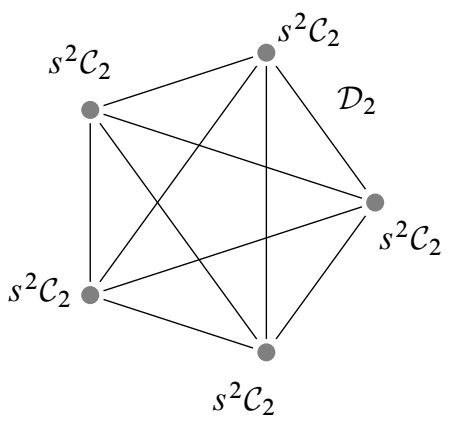

(b) $s^{3} \mathcal{C}_{2}$

Figure 1

Proposition 1.13 We have $d\left[s^{k} \mathcal{C}_{2}\right]=[k]_{2}\left[s^{k-1} \mathcal{C}_{2}\right]$.

Proof Let $T_{k+2}$ be the subgroup of $O(k+2)$ generated by reflections of the standard basis vectors. Then $s^{k} \mathcal{C}_{2}$ is the index two subgroup of orientation-preserving elements in $T_{k+2}$. The standard basis vectors provide the only points with vertex groups in the sphere quotient by $T_{k+2}$ or $s^{k} \mathcal{C}_{2}$. They are all folded with either group and have $O(k+2)$ conjugate local groups. For $s^{k} \mathcal{C}_{2}$ these local groups are $s^{k-1} \mathcal{C}_{2}$.

Lemma 1.14 Let $G$ be a finite degree- $n$ subgroup of $\mathrm{SO}(n)$. Then for every finite degree- $(n-2)$ subgroup $K$ of $\mathrm{SO}(n-2)$,

$$
\sum_{\left(H_{i}\right) \in \mathcal{S O}_{n-1, f}} \lambda_{H_{i}}(G) \lambda_{K}\left(H_{i}\right)=0 .
$$


Proof Consider the graph of those singular points in $S^{n-1} / G$ whose local group is either of degree $n-1$ or $n-2$. We will call this the orbifold graph of $S^{n-1} / G$ or of $G$. It is labelled by the local groups; see Example 1.12. We look at the subgraph of this consisting of those edges labelled $(K)$ and all vertices. The sum of the valencies of the vertices (the number of edges emanating from the vertex) in this subgraph must be even as it is twice the number of edges. For a point labelled $(H), \lambda_{K}(H)$ is the number of edges, mod two, labelled $(K)$ emanating from that point. The parity of such points is $\lambda_{H}(G)$. Thus the contribution from vertices labelled $H$ to this sum of valencies, mod two, is $\lambda_{H}(G) \lambda_{K}(H)$.

Theorem 1.15 We have $d^{2}=0$. Thus $\operatorname{im} d_{n+1} \subset \operatorname{ker} d_{n}$ and $(\beta, d)$ forms a chain complex. Hence we can form the homology groups associated with it. This homology will be denoted by $\mathcal{H}_{n}$.

Proof Since

$$
\begin{aligned}
d_{n-1} d_{n}[G] & =\sum_{(H) \in \mathcal{S O} \mathcal{O}_{n-1, f}} \lambda_{H}(G) d_{n-1}[H] \\
& =\sum_{(H) \in \mathcal{S O} \mathcal{O}_{n-1, f}}\left(\lambda_{H}(G) \sum_{(K) \in \mathcal{S} \mathcal{O}_{n-2, f}} \lambda_{K}(H)[K]\right) \\
& \left.=\sum_{(K) \in \mathcal{S} \mathcal{O}_{n-2, f}}\left(\sum_{(H) \in \mathcal{S} \mathcal{O}_{n-1, f}} \lambda_{H}(G) \lambda_{K}(H)\right)[K]\right),
\end{aligned}
$$

this follows from the previous lemma.

We will denote the homology equivalence relation by $\sim_{d}$ and the homology equivalence class of $M \in \beta_{n}$ by $[M]_{d}$.

We have that $\mathcal{H}_{0}$ and $\mathcal{H}_{1}$ are zero since $\beta_{0}=\beta_{1}=0$.

Proposition 1.16 We have $\mathcal{H}_{2}=0$.

Proof We have $\beta_{2}=\bigoplus_{k \geq 2}\left[\mathcal{C}_{k}\right]$ and $d\left[\mathcal{C}_{k}\right]=0$, so ker $d_{2}=\beta_{2}$. Since $d\left[\mathcal{D}_{k}\right]=\left[\mathcal{C}_{k}\right]$, $\operatorname{ker} d_{2}=\operatorname{im} d_{3}$.

Proposition 1.17 We have $\mathcal{H}_{3}=0$.

Proof We apply Proposition 1.11. Let $\sigma \in \operatorname{ker} d_{3}$. If $\left[\mathcal{D}_{k}\right]$ is a nontrivial summand of $\sigma$, then since $d\left[\mathcal{D}_{k}\right]=\left[\mathcal{C}_{k}\right], \sigma$ must additionally contain a nondihedral summand. Hence $k \leq 5$. Because $d[\mathcal{T}]=\left[\mathcal{C}_{2}\right]$, we see that the minimal (fewest summands) 
element of $\operatorname{ker} d_{3}$ having $[\mathcal{T}]$ as a summand is $[\mathcal{T}]+\left[\mathcal{D}_{2}\right]$. With $d[\mathcal{O}]=\left[\mathcal{C}_{2}\right]+\left[\mathcal{C}_{3}\right]+$ $\left[\mathcal{C}_{4}\right]$ and $d[\mathcal{I}]=\left[\mathcal{C}_{2}\right]+\left[\mathcal{C}_{3}\right]+\left[\mathcal{C}_{5}\right]$, minimal elements of ker $d_{3}$ containing these are $[\mathcal{M}]+\left[\mathcal{D}_{3}\right]+\left[\mathcal{D}_{4}\right]+[\mathcal{O}]$ and $[\mathcal{M}]+\left[\mathcal{D}_{3}\right]+\left[\mathcal{D}_{5}\right]+[\mathcal{I}]$, where $\mathcal{M}$ is either $\mathcal{D}_{2}$ or $\mathcal{T}$, or $\left[\mathcal{D}_{4}\right]+[\mathcal{O}]+\left[\mathcal{D}_{5}\right]+[\mathcal{I}]$. The latter element of ker $d_{3}$ is the sum of the former two. It follows that $\operatorname{ker} d_{3}$ is generated by (i), (ii) and (iii) below. Additionally $[\mathcal{T}]+\left[\mathcal{D}_{3}\right]+\left[\mathcal{D}_{4}\right]+[\mathcal{K}], \mathcal{K}=\mathcal{O}$ or $\mathcal{I}$ are obtained by adding (i), (ii) or (iii):

(i) $[\mathcal{T}]+\left[\mathcal{D}_{2}\right]$.

(ii) $\left[\mathcal{D}_{2}\right]+\left[\mathcal{D}_{3}\right]+\left[\mathcal{D}_{4}\right]+[\mathcal{O}]$.

(iii) $\left[\mathcal{D}_{2}\right]+\left[\mathcal{D}_{3}\right]+\left[\mathcal{D}_{5}\right]+[\mathcal{I}]$.

From Proposition 2.20 or the author [5, Theorem 3.2], we conclude that these elements are in turn $d_{4}\left[G \ltimes-\mathrm{id}_{4}\right]$, where $G=[\mathcal{T}],[\mathcal{O}],[\mathcal{I}]$, respectively.

\section{Algebraic properties of $d$}

Suppose $G$ as a linear group is the direct sum $\left(\phi_{1} \oplus \phi_{2}\right)(G)$ of the representations $\phi_{1}$ and $\phi_{2}$ of the abstract group $G$ with degrees $n_{1}$ and $n_{2}$. Let $\pi_{i}, i=1,2$, be the projections $O\left(n_{1}\right) \times O\left(n_{2}\right) \rightarrow O\left(n_{i}\right), i=1,2$. Let $K_{1}=\operatorname{ker} \pi_{2 \mid G}, K_{2}=\operatorname{ker} \pi_{1 \mid G}$. We have that $K_{i}$ is isomorphic to a subgroup of $\phi_{i}(G)$ which we also denote by $K_{i}$. The product $K_{1} \times K_{2}$ is a linear subgroup of $G$, and it is the largest linear product in $G$. The projection $\pi_{i \mid G}$ factors through $\phi_{i}(G)$ and we get the isomorphisms

$$
G / K_{2} \cong \operatorname{im} \pi_{1 \mid G} \cong \phi_{1}(G), \quad G / K_{1} \cong \operatorname{im} \pi_{2 \mid G} \cong \phi_{2}(G) .
$$

Using the third isomorphism theorem, we have that $G /\left(K_{1} \times K_{2}\right) \cong \phi_{1}(G) / K_{1}$ $\cong \phi_{2}(G) / K_{2}$. We denote this common quotient group as $L$. The quotient of $\mathbb{R}^{n_{1}+n_{2}}$ by $G$ can be factored as $\mathbb{R}^{n_{1}+n_{2}} \rightarrow \mathbb{R}^{n_{1}} / K_{1} \times \mathbb{R}^{n_{2}} / K_{2} \rightarrow \mathbb{R}^{n_{1}+n_{2}} / G$. The second quotient comes from the diagonal action of $L$, where the choices of representatives act linearly on each $\mathbb{R}^{n_{i}}$. The above argument follows along that used for determining the subgroups of $S^{3} \times S^{3}$ in Du Val [6, Chapter 3].

We use this sequence of quotients in the following decomposition of $S^{n-1} / G$, which will aid in the calculation of $d$ of the direct sum:

$$
S^{n-1} / G=\left(S^{n_{1}-1} / K_{1} \times_{L} D^{n_{2}} / K_{2}\right) \cup \sim_{i}\left(D^{n_{1}} / K_{1} \times_{L} S^{n_{2}-1} / K_{2}\right) .
$$

Here $\sim_{i}$ indicates the two products are sewn together along $S^{n_{1}-1} / K_{1} \times{ }_{L} S^{n_{2}-1} / K_{2}$. For $F$ a subset of $\phi_{1}(G)$, respectively of $\phi_{2}(G)$, let $F^{*}$ denote the corresponding subset in the linear group $G$ containing $K_{2}$, respectively $K_{1}$ in (1). Let $c c_{i}$ be the conjugacy classes $\left(F_{i}\right)_{\phi_{i}(G)} \in \mathcal{L}\left(\phi_{i}(G)\right)$ for which the other summand $\phi_{j}\left(F_{i}^{*}\right)$ has full degree and hence the corresponding subgroup $F_{i}^{*}$ of $G$ is a vertex group. 
By considering the two products in the union in (2), we obtain the following.

Proposition 2.1 We have

$$
d[G]=\sum_{\left(F_{1}\right)_{\phi_{1}(G)} \in c c_{1}} n_{F_{1}, \phi_{1}(G)}\left[F_{1}^{*}\right]+\sum_{\left(F_{2}\right)_{\phi_{2}(G)} \in c c_{2}} n_{F_{2}, \phi_{2}(G)}\left[F_{2}^{*}\right] .
$$

Proof The first and second summands correspond to vertex groups of the first and second products of (2). By the third isomorphism theorem, we need only consider conjugacy classes and normalizers in $\phi_{i}(G)$ instead of $G$. However we cannot further group according to $O\left(n_{i}\right)$ conjugacy classes in these sums as those classes may not be preserved in pulling back to $G$.

Proposition 2.2 (i) If $G$ has no direct summands of real type, then $d[G]=0$.

(ii) For any faithful representation $\phi$ of a finite group $G$ into $O(n)$ and $k>1$ with $\phi(G)^{\oplus k} \in \mathrm{SO}(n k), d\left[\phi(G)^{\oplus k}\right]=0$.

(iii) We can decompose $G$ as $\phi_{1}(G) \oplus \phi_{2}(G)$ where the first summand has only real, nonrepeating (as in (ii)) direct summands and the second has the repeating or complex or symplectic summands. Then $d[G]=\sum_{\left(F_{1}\right)_{\phi_{1}(G)} \in c c_{1}} n_{F_{1}, \phi_{1}(G)}\left[F_{1}^{*}\right]$.

Proof (i) This is because $G$ and all its subgroups are of even degree, and so no subgroup is of codegree 1 .

(ii) No subgroup has codegree 1 .

(iii) We apply Proposition 2.1 and (i) and (ii).

Using the standard inclusion of $\mathrm{SO}\left(n_{1}\right) \times \mathrm{SO}\left(n_{2}\right)$ in $\mathrm{SO}\left(n_{1}+n_{2}\right)$, we define a product in $\beta$ via $\left[G_{1}\right] \times\left[G_{2}\right]=\left[G_{1} \times G_{2}\right]$ for groups and then extend this to sums of such elements.

Corollary 2.3 $d$ is a derivation.

Proof It suffices to check this on basis elements which are products. If $G$ is a linear product, then $G=K_{1} \times K_{2}$ and $L$ is trivial in the discussion preceding Proposition 2.1. Equation (2) above becomes

$$
S^{n-1} / G=\left(S^{n_{1}-1} / K_{1} \times D^{n_{2}} / K_{2}\right) \cup \sim_{i}\left(D^{n_{1}} / K_{1} \times S^{n_{2}-1} / K_{2}\right) .
$$

A vertex group in the first product above must be of the form $H_{1} \times K_{2}$, where $H_{1}$ is a vertex group of $K_{1}$, and a similar result holds for the second product. Furthermore 
by considering the portion $S^{n_{1}-1} / K_{1} \times D^{n_{2}} / K_{2}$, we see that $\lambda_{H_{1} \times K_{2}}\left(K_{1} \times K_{2}\right)=$ $\lambda_{H_{1}}\left(K_{1}\right)$. Thus we have

$$
\begin{aligned}
d\left[K_{1} \times K_{2}\right] & =\sum_{\mathcal{S} \mathcal{O}_{n-1, f}} \lambda_{H}\left(K_{1} \times K_{2}\right)[H] \\
& =\sum_{\mathcal{S}_{1}} \lambda_{H_{1} \times K_{2}}\left(K_{1} \times K_{2}\right)\left[H_{1} \times K_{2}\right]+\sum_{\mathcal{S}_{2}} \lambda_{K_{1} \times H_{2}}\left(K_{1} \times K_{2}\right)\left[K_{1} \times H_{2}\right] \\
& =\left(\left(\sum_{\mathcal{S}_{1}} \lambda_{H_{1}}\left(K_{1}\right)\left[H_{1}\right]\right) \times\left[K_{2}\right]\right)+\left(\left[K_{1}\right] \times\left(\sum_{\mathcal{S}_{2}} \lambda_{H_{2}}\left(K_{2}\right)\left[H_{2}\right]\right)\right) \\
& =\left(d_{n_{1}}\left[K_{1}\right]\right) \times\left[K_{2}\right]+\left[K_{1}\right] \times\left(d_{n_{2}}\left[K_{2}\right]\right) .
\end{aligned}
$$

Here $\mathcal{S}_{i}$ stands for $\mathcal{S O}_{n_{i}}, f$. Since we are working with $Z_{2}$, the usual power of -1 in front of the second summand is irrelevant.

The following propositions are immediate consequences of Corollary 2.3.

Proposition 2.4 We have $d[G \times G]=0$.

Proposition 2.5 If $G=G_{1} \times G_{2}$ where $G_{2}$ has no direct summand of real type, then $d[G]=d\left[G_{1}\right] \times\left[G_{2}\right]$.

Definition 2.6 For $(G) \in \mathcal{S O}_{n, f}$, let $N(G)_{2}^{-}$be the set of orientation-reversing linear automorphisms $\alpha$ of $G$ in $O(n)$ with $\alpha^{2} \in G$. Let $\beta_{n}^{-}$, respectively $\beta_{n}^{+}$, denote the subspace of $\beta_{n}$ generated by classes of groups $G$ for which $N(G)_{2}^{-}$is nonempty, respectively empty.

Proposition 2.7 We have $\operatorname{im} d_{n+1} \subseteq \beta_{n}^{-}$. Hence $\operatorname{ker} d_{n} \cap \beta_{n}^{+}$is isomorphic to a subgroup of $\mathcal{H}_{n}$.

Proof If $[H]$ is a nonzero summand of $d[G], \lambda_{H}(G) \neq 0$, and so by Proposition 1.7 $\mathcal{L}(G, H)_{-}$is nonempty. Thus assume $H<G$ and $\alpha \in G$ folds the line fixed by $H$. We can also assume $H<\mathrm{SO}(n)<\mathrm{SO}(n+1)$, in which case $\alpha=\left(\alpha_{1},-1\right)$ with $\alpha_{1} \in O(n)$ the orientation-reversing automorphism of $H$.

Proposition 2.8 We have that $\mathcal{H}_{4}$ is nontrivial.

Proof Let $G=\left\langle\left[\begin{array}{cc}e^{(2 \pi i) / m} & 0 \\ 0 & e^{(2 \pi i) / m}\end{array}\right]\right\rangle$. Here we are considering the generator of $G$ as an element of $O(4)$ via the standard inclusion of $U(2)$ in $O(4)$. If $m>2,[G] \in$ ker $d_{4} \cap \beta_{4}^{+}$, and we can apply Proposition 2.7. 
Proposition 2.9 We have $\beta_{2 k+1}^{-}=\beta_{2 k+1}$.

Proof We have that $-\mathrm{id}_{n}$ commutes with any group of degree $n$.

Definition 2.10 Suppose $\alpha \in N(G)_{2}^{-}$. Let $\widehat{\alpha}=(\alpha,-1) \in \operatorname{SO}(n+1)$ and $s_{\alpha}(G)=$ $\langle G, \hat{\alpha}\rangle$. Then $S^{n} / s_{\alpha}(G)$ is the quotient of $S^{n}$ by $G$ followed by the folding by $\hat{\alpha}$.

One goal in considering $s_{\alpha}(G)$ is to replace $G$ with simpler vertex groups. Hence we would like to relate the parity of the vertex groups of $G$ to those of $s_{\alpha}(G)$. Suppressing the choice of $\alpha$ for $G$, we have $s: \beta_{n}^{-} \rightarrow \beta_{n+1}^{-}$. (The image is in $\beta_{n+1}^{-}$since the diagonal matrix $\left(\mathrm{id}_{n},-1\right)$ commutes with $s_{\alpha}(G)$.) A natural question is: what is $d s-s d$ ? Theorem 2.12 below gives a partial answer to this. Here we are interpreting $s d$ with $s$ being distributed past the sum for $d$ and applied in a natural manner to each summand; see Corollary 2.18. As in Proposition 2.1, we cannot necessarily group the $O(n)$ conjugates of vertex subgroups of $G$.

Definition 2.11 We have that $\alpha$ acts on the conjugacy classes of $G$ and hence on $\mathcal{L}(G)$. Let $\mathcal{L}(G)^{\alpha}$ denote those elements which are invariant under $\alpha$. In addition, let $\mathcal{L}(G)_{ \pm}^{\alpha}=$ $\mathcal{L}(G)^{\alpha} \cap \mathcal{L}(G)_{ \pm}$.

Theorem 2.12 We have

$$
d\left[s_{\alpha}(G)\right]=[G]+\sum_{\mathcal{L}(G)^{\alpha}}\left[s_{\alpha_{H, G}}(H)\right]+A .
$$

Furthermore, we have that $\widehat{\alpha_{H, G}}$ is either $\alpha$ or $\alpha \gamma_{H}$ where $\gamma_{H}$ is an element of $G$ which folds the fixed line of $H$. Here $A$ consists of the summands which are $Z_{2}$ or $Z_{2}$-extensions of isotropy groups of $G$ of degree less than $n-1$.

For each $(H) \in \mathcal{G}^{v}(G)$, we want to look more closely at that portion of the orbifold graph of $s_{\alpha} G$ labelled by $(H)$; see the proof of Lemma 1.14 for the definition of the orbifold graph of a group $D$. For a given representative $(H)_{G} \in \mathcal{L}(G)$, the contribution by the fixed point set of $(H)_{s_{\alpha}(G)}$ to this subgraph is specified by Proposition 2.15.

Definition 2.13 For $D$ a finite degree- $n$ subgroup of $\mathrm{SO}(n)$ and $K<D$, the $(K)_{D^{-}}$ singular set is the closure of those points in $S^{n-1} / D$ whose isotropy group is conjugate to $K$ in $D$. If the degree of $K$ is $n-2$, the $(K)_{D}$-singular set is a graph whose edges are labelled by $(K)$ and whose vertices are labelled by elements of $\mathcal{G}^{v}(D)$. 
Proof of Theorem 2.12 In $S^{n} / s_{\alpha}(G)$ the image of the point in $S^{n}$ with last coordinate \pm 1 contributes one singular vertex point labelled $G$. All other singular vertex points occur at the images of points $(v, 0), v \in S^{n-1}$ and become vertex points only when we quotient $S^{n} / G$ by $\hat{\alpha}$. These points have vertex groups which are $Z_{2}$ or $Z_{2}$-extensions of isotropy groups of $G$. The orbifold graph for $S^{n} / s_{\alpha}(G)$ is the quotient of that for $S^{n} / G$ with possible vertices and edges added at $x_{n+1}=0$.

Now suppose $(H)_{G} \in \mathcal{L}(G)$. We consider the portion of the orbifold graph of $S^{n} / s_{\alpha}(G)$ given by the $(H)_{s_{\alpha}(G)}$-singular set. By Proposition 2.15 this singular set looks like one of the four diagrams in Figure 2 and is (c) if and only if $(H)_{G} \in \mathcal{L}(G)_{-}^{\alpha}$. This is the only case where an extension of $(H)_{s_{\alpha}(G)}$ contributes exactly one singular point to $S^{n} / s_{\alpha}(G)$ at $x_{n+1}=0$. Furthermore that extension is $s_{\alpha_{H, G}}(H)$, where $\widehat{\alpha_{H, G}}$ is either $\alpha$ or $\alpha \gamma_{H}$, depending on whether $\alpha v= \pm v$.

It may not be true that $\left(s_{\alpha_{H, G}}(H)\right)=\left(s_{\alpha_{H^{\prime}, G}}\left(H^{\prime}\right)\right)$ when $(H)=\left(H^{\prime}\right)$, so we cannot necessarily group according to $O(n)$ conjugacy in the sum for $d s[G]$.

Remark 2.14 The portion of our orbifold graph with edges labelled $(H)$ which arises from $\mathcal{L}(G, H)$ may consist of a wedge of some combination of the graphs given in Figure 2, all joined at the vertex point labelled $(G)$, since the action of $\alpha$ on the various representatives of $(H)$ in $\mathcal{L}(G)$ may differ. Additionally there may be new edges labelled $(H)$ occurring at $x_{n+1}=0$; see Example 2.16 and the associated Figure 3.

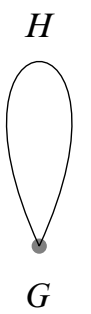

(a)

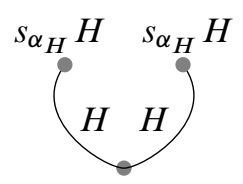

G

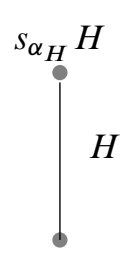

$G$

(c)

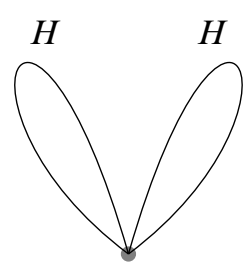

$G$

(d)

Figure 2

Proposition 2.15 Suppose that $(H)_{G} \in \mathcal{L}(G)$. Then the $(H)_{s_{\alpha}(G)}$-singular set in $S^{n} / s_{\alpha}(G)$ is one of the four labelled graphs in Figure 2. These graphs correspond in order to:

(a) $(H)_{G} \in \mathcal{L}(G)_{+}^{\alpha}$ and $\alpha v=-v$, or $(H)_{G} \in \mathcal{L}(G)_{-}-\mathcal{L}(G)^{\alpha}$.

(b) $(H)_{G} \in \mathcal{L}(G)_{+}^{\alpha}$ and $\alpha v=v$.

(c) $(H)_{G} \in \mathcal{L}(G)_{-}^{\alpha}$.

(d) $(H)_{G} \in \mathcal{L}(G)_{+}-\mathcal{L}(G)^{\alpha}$. 
Proof If $(H)_{G} \in \mathcal{L}(G)$ and $H$ is the isotropy group for $\pm v \in S^{n-1}$, it fixes the circle $\left( \pm \sqrt{1-t^{2}} v, t\right)$ in $S^{n}$. The quotient of this circle in $S^{n} / G$ is either a circle or an arc labelled $(H)$ with opposite points (the image of $(0, \pm 1)$ ) labelled $G$, according to whether $(H)_{G} \in \mathcal{L}(G)_{+}$or $(H)_{G} \in \mathcal{L}(G)_{-}$.

We now look at the quotient of $S^{n} / G$ by $\widehat{\alpha}$.

Consider first the case where $(H)_{G} \in \mathcal{L}(G)^{\alpha}$ and thus $\hat{\alpha}$ sends the circle or arc to itself. For the circle we get (a) or (b), depending on whether $\alpha v=\mp v$. For $\alpha v=v$ in (b) we have $\widehat{\alpha_{H, G}}=\alpha$. For the arc we get (c) and $\widehat{\alpha_{H, G}}=\alpha$ or $\alpha \gamma_{H}$, according to whether $\alpha v= \pm v$.

If $(H)_{G} \notin \mathcal{L}(G)^{\alpha}$, we consider the two circles or two arcs in $S^{n} / G$ coming from the fixed point sets for $(H)_{G}$ and $\left(H^{\alpha}\right)_{G}$. When $N_{G}(H)=H$, we have the two circles, which are then identified by $\hat{\alpha}$ to get graph (d). For $N_{G}(H) \neq H$, we get two arcs, which are then identified by $\hat{\alpha}$ giving us (a).

Example 2.16 Let $\alpha=-\mathrm{id}_{3}$. The orbifold graph for $G=s_{\alpha} \mathcal{D}_{k}$ is given below as (a) for $k$ even or (b) for $k$ odd. We have $\mathcal{L}\left(\mathcal{D}_{k}\right)^{\alpha}=\mathcal{L}\left(\mathcal{D}_{k}\right)$. The graph for $\left(\mathcal{C}_{j}\right)_{s_{\alpha} \mathcal{D}_{k}}$, $j=k$, or $j=2$ when $k$ is even, is given in (c). When $j=2$ and $k$ is odd, the graph is as in (d). The full graph for $\left(\mathcal{C}_{2}\right)$ (as in Figure 3(a) and (b)) is larger and involves symmetry arising from the fact that $G=s^{2} \mathcal{C}_{k}$. In the figure the open circles indicate the image of the point at $(0, \pm 1)$ and unlabelled edges have local group $\mathcal{C}_{2}$.

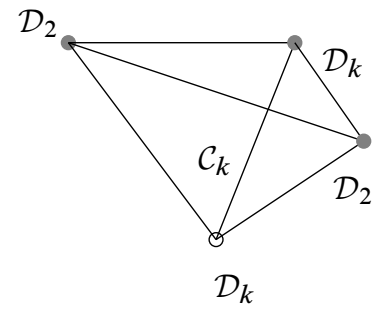

(a)

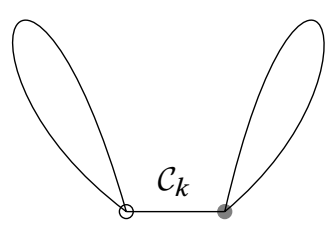

$\mathcal{D}_{k} \quad \mathcal{D}_{k}$

(b)

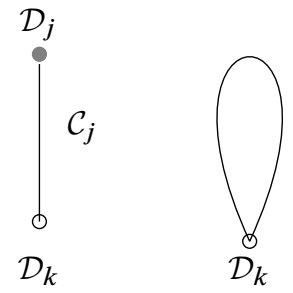

(c)

(d)

Figure 3

Proposition 2.17 Suppose $\alpha \in N(G)_{2}^{-}$. Then $\lambda_{H}(G)=\left[\left|\mathcal{L}(G)_{-}^{\alpha} \cap \mathcal{L}(G, H)\right|\right]_{2}$.

Proof We look at $(v, 0)$ in $S^{n-1} / G \subset S^{n} / G$ in the proof for Proposition 2.15 and note that when $(K)_{G} \in \mathcal{L}(G, H)$ it only contributes to $\lambda_{H}(G)$ nontrivially when we are in case (c). 
Corollary 2.18 The formula in Theorem 2.12 would give us $d s_{\alpha}-s_{\alpha} d=$ id if:

(i) $A=0$.

(ii) $s_{\alpha_{H^{\prime}, G}}\left(H^{\prime}\right) \sim O(n) s_{\alpha_{H, G}}(H)$ for each $\left(H^{\prime}\right)_{G} \in \mathcal{L}(G, H)$.

Proof For $d G=\sum \lambda_{H}(G)[H]$, it makes sense by (ii) to group according to conjugacy in $O(n)$ and have $s_{\alpha} d G=\sum \lambda_{H}(G)\left[s_{\alpha_{H, G}} H\right]$. Further, by Proposition 2.17,

$$
\begin{aligned}
\sum_{(K)_{G} \in \mathcal{L}(G)^{\alpha}}\left[s_{\alpha_{K, G}}(K)\right] & =\sum_{(H) \in \mathcal{S} \mathcal{O}_{n, f}} \sum_{(K)_{G} \in \mathcal{L}(G)^{\alpha} \cap \mathcal{L}(G, H)}\left[s_{\alpha_{K, G}}(K)\right] \\
& =\sum_{(H) \in \mathcal{S} \mathcal{O}_{n, f}} \lambda_{H}(G)\left[s_{\alpha_{H, G}}(H)\right] .
\end{aligned}
$$

This completes the proof.

Corollary 2.19 In the case where $\alpha=-\mathrm{id}_{n}$,

$$
d\left[s_{\alpha}(G)\right]=[G]+\sum_{\mathcal{L}(G)_{-}}\left[s_{-\gamma_{H}}(H)\right]+A
$$

and all singular sets in $S^{n} / s_{\alpha}(G)$ for $H$ a vertex group for $G$ look like either (a) or (c) in Figure 2, depending on whether $N_{G}(H)=H$ or not.

Proof We have that $\alpha$ commutes with $G$, so $\mathcal{L}(G)^{\alpha}=\mathcal{L}(G)$ and $\alpha v=-v$. Hence we are in case (a) or (c) of Proposition 2.15.

We illustrate these results with an application to degree-three groups. This is a formalization in our current notation of a proof we gave in [5].

Proposition 2.20 For $\alpha=-\mathrm{id}_{3}, d\left[s_{\alpha} \mathcal{I}\right]=[\mathcal{I}]+\left[\mathcal{D}_{2}\right]+\left[\mathcal{D}_{3}\right]+\left[\mathcal{D}_{5}\right], d\left[s_{\alpha} \mathcal{O}\right]=[\mathcal{O}]+$ $\left[\mathcal{D}_{2}\right]+\left[\mathcal{D}_{3}\right]+\left[\mathcal{D}_{4}\right], d\left[s_{\alpha} \mathcal{T}\right]=[\mathcal{T}]+\left[\mathcal{D}_{2}\right]$, and $d\left[s_{\alpha} \mathcal{D}_{k}\right]=0$.

Proof When $\mathcal{K}$ is a finite subgroup of $\mathrm{SO}(3)$, its nontrivial subgroups have degree 2, so $A=0$ in Corollary 2.18. Also for any $\tau \in N\left(\mathcal{C}_{k}\right)_{2}^{-}, s_{\tau} \mathcal{C}_{k}$ is conjugate to $\mathcal{D}_{k}$ and so condition (ii) of that corollary also holds. Hence $d\left[s_{\alpha}(\mathcal{K})\right]=[\mathcal{K}]+s d[\mathcal{K}]$. Applying Proposition 1.11 for $d[\mathcal{K}]$ and the fact that $\left(s \mathcal{C}_{j}\right)=\left(\mathcal{D}_{j}\right)$, we get our result.

Definition 2.21 Let $\beta_{n, 0}$ be generated by those $[G] \in \beta_{n}$ such that $G$ does not have $Z_{2}$ as a direct summand. For $k>0$ we inductively define $\beta_{n, k}$ to be generated by those $[G] \in \beta_{n}$ such that $G=\phi_{1}(G) \oplus \phi_{2}(G)$, where $\phi_{1}(G)$ has an index-one or index-two subgroup in $\beta_{j, k-1}$ for some $j<n, G / \phi_{1}(G)=Z_{2}$, and $\phi_{2}(G)=\left\langle-\mathrm{id}_{n-j}\right\rangle$. Also let $\beta_{n, k}^{ \pm}=\beta_{n, k} \cap \beta_{n}^{ \pm}$. Our convention is that $\left\langle-\mathrm{id}_{n}\right\rangle, n \geq 0$ and even, is in $\beta_{n, 1}$. 
Example 2.22 We have $\beta_{3,0}=[\mathcal{I}] \oplus[\mathcal{O}] \oplus[\mathcal{T}]$ and $\beta_{3,1}=\bigoplus_{k \geq 3}\left[\mathcal{D}_{k}\right]$. $\beta_{3,2}=\left[\mathcal{D}_{2}\right]$.

Proposition 2.23 (i) All irreducible groups in $\beta_{n}$ fall in $\beta_{n, 0}$.

(ii) With the exception of $Z_{2}$, the representation of any simple group is in $\beta_{n, 0}$.

(iii) We have that $\beta_{n, k}=\beta_{n, k}^{-}$for $k>0$.

Proof (i) If $[G] \in \beta_{n, k}$ with $k>0, G$ has a direct summand of $Z_{2}$.

(ii) All representations of simple groups are faithful, and so unless a simple group is $Z_{2}$, it cannot have a $Z_{2}$ direct summand.

(iii) We can assume the $Z_{2}$ summand acts by -1 on $x_{n}$, and then we have that (id $\left.{ }_{n-1},-1\right)$ commutes with $G$.

Corollary 2.24 Suppose $[G] \in \beta_{n, 0}^{-}$and $\alpha \in N(G)_{2}^{-}$. Then:

(i) We have that $\lambda_{G}\left(s_{\alpha}(G)\right)=1$, and hence $d\left[s_{\alpha}(G)\right] \neq 0$.

(ii) If $M \in \operatorname{ker} d_{n+1}$ with $\left[s_{\alpha}(G)\right]$ as a nontrivial summand, then there is another summand $[L]$ of $M$ with $\lambda_{G}(L)=1$, and either $[L] \in \beta_{n+1,0}$ or $(L)=\left(s_{\tau}(G)\right)$ for some $\tau \in N(G)_{2}^{-}$with $\left(s_{\tau}(G)\right) \neq\left(s_{\alpha}(G)\right)$.

Proof Statement (i) is verified by applying Theorem 2.12 and noting that $[G] \neq$ $\left[s_{\alpha_{H}}(H)\right]$ nor is it a summand of $A$.

For (ii), Proposition 2.1 and Theorem 2.12 tell us that the only way that $[G]$ is a nontrivial summand of $d[L]$ for $[L] \in \beta_{n+1, k}$ with $k>0$ is if $(L)=\left(s_{\tau}(G)\right)$ for some $\tau \in N(G)_{2}^{-}$. In that case, since $\left[s_{\alpha}(G)\right]$ is a nontrivial summand of $M,\left(s_{\alpha}(G)\right) \neq\left(s_{\tau}(G)\right)$.

Definition 2.25 Define an equivalence relation $\sim_{b}$ on $N(G)_{2}^{-}$by setting $\alpha \sim_{b} \alpha^{\prime}$ if $\left(s_{\alpha}(G)\right)=\left(s_{\alpha^{\prime}}(G)\right)$.

Definition 2.26 Let $\beta_{n, 1, j}$ be generated by those elements of $[K] \in \beta_{n, 1}$ for which the maximal $Z_{2}$ summand has degree $j$. Let $\beta_{n, 1,1}^{*}$ be generated by those $\left[s_{\alpha}(G)\right]$, $[G] \in \beta_{n-1,0}^{-}$for which $\left|\left(N(G)_{2}^{-} / \sim_{b}\right)\right|>1$.

Corollary 2.27 Let $M \in \beta_{n}$. Then:

(i) $M \sim_{d} M^{\prime}$ where $M^{\prime} \in \beta_{n}^{+} \oplus \beta_{n, k>0}$.

(ii) If $M \in \operatorname{ker} d_{n} \cap \beta_{n, k>0}, M \in \beta_{n, 1,1}^{*} \oplus \beta_{n, 1, j>1} \oplus \beta_{n, k>1}$.

(iii) If $n$ is odd and $M \in \operatorname{ker} d_{n}, M \sim_{d} M^{\prime}$ where $M^{\prime} \in \beta_{n, 1,1}^{*} \oplus \beta_{n, 1, j>1} \oplus \beta_{n, k>1}$.

Here $\beta_{n, k>l}=\bigoplus_{k>l} \beta_{n, k}$ and similarly for $\beta_{n, 1, j>l}$. 
Proof (i) We can write $M$ as $M_{+}+M_{-}$where $M_{ \pm} \in \beta_{n}^{ \pm}$. We then apply Theorem 2.12 to those summand of $M_{-}$which are in $\beta_{n, 0}^{-}$.

(ii) We use Corollary 2.24 for those summands of $M$ which are in $\beta_{n, 1,1}$ and hence of the form $s_{\alpha}(G)$ for $[G] \in \beta_{n-1,0}^{-}$. Since $M$ has no summands from $\beta_{n, 0}$, we have our result.

(iii) When $n$ is odd, $\beta_{n}^{+}$(which is $\beta_{n, 0}^{+}$) is trivial and so (i) implies $M \sim_{d} M^{\prime}$ with $M^{\prime} \in \operatorname{ker} d_{n} \cap \beta_{n, k>0}$, and then we apply (ii).

\section{The relationship of $d$ and $\mathcal{H}_{n}$ to orbifolds and oriented orbifold cobordism}

We now collect some definitions and constructions relevant to oriented orbifold cobordisms. Most of these are from a previous paper [5] as well as the many other references on orbifolds, including Adem, Leida and Ruan [1]. We are restricting our attention to effective, smooth orbifolds.

An $n$-orbifold $Q$ is built out of charts $C=(U, H, \bar{U}, \pi)$, where $U$ is an open subset of the underlying topological space $|Q|, \bar{U}$ is an open subspace of $\mathbb{R}^{n}, H$ is a finite group acting effectively on $\bar{U}$ and $\pi: \bar{U} \rightarrow U$ is an open onto map which factors as $h p_{U}$ with $p_{U}$ the orbit map and $h$ a homeomorphism from $\bar{U} / H$ to $U$. The local group at a point $x \in U \subset|Q|$ is the subgroup of $H$ which fixes an element of $\pi^{-1}(x)$. One has the notion of an overlap map between two compatible charts, $C_{1}$ and $C_{2}$. This is a local diffeomorphism between some open subsets of $\bar{U}_{1}$ and $\bar{U}_{2}$ which commutes with $\pi_{1}$ and $\pi_{2}$. An orbifold is then defined by a maximal atlas of compatible charts. Using the differential of $H$ the local group at a point is well defined up to conjugacy as a subgroup of $\operatorname{Gl}(n, R)$. Once a Riemannian metric is placed on the orbifold this local group is well defined up to conjugacy in $O(n)$. For a paracompact orbifold we can assume such a metric is given. We denote by $\mathcal{G}(Q)$ the set of $O(n)$ conjugacy classes of local groups of the orbifold $Q$. If all the local groups of an orbifold are subgroups of $\mathrm{SO}(n)$, the orbifold is said to be locally orientable. An orientation for a locally orientable orbifold is given by specifying an atlas whose charts are oriented and the overlap maps are orientation-preserving [5].

For $W$ an oriented orbifold with boundary, let $\partial_{o} W$ denote the orbifold boundary of $W$ with the standard induced orientation. Two oriented orbifolds $Q_{1}, Q_{2}$ are oriented orbifold cobordant, written $Q 1 \sim_{o} Q 2$, if $Q 1 \amalg-Q 2=\partial_{o} W$ for some $W$. Let $\Omega_{n \text {,orb }}$ be the group of oriented orbifold cobordism classes of compact $n$-dimensional oriented orbifolds as defined in a previous paper [4]. 
For an isolated singular point $x$ in the oriented orbifold $Q$, once one chooses the chart with the standard orientation, the vertex group is well defined up to conjugacy class in $\mathrm{SO}(n)$, not necessarily just $O(n)$; we will call it the oriented vertex group of the point. If $H$ is an oriented vertex group for $Q$ at $x$, a neighborhood of $x$ in $Q$ is then oriented orbifold diffeomorphic (see [4, page 300]) to $\dot{D}^{n} / H$, where $\dot{D}^{n}$ is the open disc with standard orientation. If $\alpha \in O(n)$ has determinant $-1, \dot{D}^{n} / H$ is oriented orbifold diffeomorphic to $-\dot{D}^{n} /\left(\alpha H \alpha^{-1}\right)$ via the natural quotient map $\bar{\alpha}$.

If $[H] \in \beta_{n}^{-}$and $\gamma$ is an orientation-reversing automorphism of $H$, we have that $\dot{D}^{n} / H$ is orientably diffeomorphic to $\dot{D}^{n} /\left(\alpha \gamma H \gamma^{-1} \alpha^{-1}\right)$, which in turn is identical to $\dot{D}^{n} /\left(\alpha H \alpha^{-1}\right)$. Thus in this case, the oriented vertex group is well defined up to conjugacy in $O(n)$.

In our paper on rational oriented orbifold cobordisms [4], rational $H$-characteristic numbers are defined for an oriented $n$-orbifold $Q$ and any finite subgroup of $\mathrm{SO}(n)$. In the following definition we give the formal interpretation of what these numbers are for degree- $n$ groups.

Definition 3.1 For $(H) \in \mathcal{S O}_{n, f}$ the rational $H$-characteristic numbers of the compact, oriented, $n$-orbifold $Q$ are defined as follows. Let $Q_{H}=\left\{x_{1}, x_{2}, \ldots, x_{k}\right\}$ be the points in $Q$ with vertex group $H$ (not necessarily oriented vertex group.) Then the $H$-characteristic numbers of $Q$ are defined to be $\sum\left\langle f_{i}^{*} y \cup p_{0}\left(x_{i}\right),\left[x_{i}\right]\right\rangle$, where $y \in H^{0}\left(B N_{O(n)}(H) / H ; \hat{\boldsymbol{Q}}\right)$ and $f_{i}$ is a classifying map for the (trivial) normal bundle to $x_{i}$. Also $\widehat{\boldsymbol{Q}}$ denotes the coefficient system defined by the orientation of the canonical $\left(\mathbb{R}^{n} / H, N_{O(n)}(H) / H\right)$-bundle over the classifying space $B\left(N_{O(n)}(H) / H\right)$.

When $[H] \in \beta_{n}^{-}$, these numbers are zero since $H^{*}\left(B N_{O(n)}(H) / H ; \hat{\boldsymbol{Q}}\right)$ consists of the -1 eigenvectors of the natural involution on $H^{*}\left(B N_{\mathrm{SO}(n)}(H) / H ; \boldsymbol{Q}\right)$ and $H^{0}\left(B N_{\mathrm{SO}(n)}(H) / H ; \boldsymbol{Q}\right)$ is invariant under this involution.

If $[H] \in \beta_{n}^{+}$, we need to specify the sign of $\left[x_{i}\right]$. For those points whose oriented vertex group is $H$, the points are given a positive orientation. Those points whose oriented vertex group is only conjugate to $H$ in $O(n)-\mathrm{SO}(n)$ are given a negative orientation. Thus in this case, these numbers are zero precisely when the number of points with oriented vertex group $H$ is the same as that of those with vertex group conjugate to $H$ in $O(n)-\mathrm{SO}(n)$.

Let $[H] \in \beta_{n}$. Define $\mathcal{W}(H)=D^{n} / H \times I$, which is an oriented orbifold with boundary once one straightens the angle [4]. The oriented vertex group at $(\overline{0}, 0)$ is $H$. At $(\overline{0}, 1)$, with the induced boundary orientation, the boundary has a neighborhood which is oriented orbifold diffeomorphic to $-\dot{D}^{n} / H$, which in turn is oriented orbifold 
diffeomorphic to $\dot{D}^{n} /\left(\alpha H \alpha^{-1}\right)$ for any $\alpha \in O(n)-\mathrm{SO}(n)$. Hence the oriented vertex group for the boundary at $(\overline{0}, 1)$ is $\alpha H \alpha^{-1}$. The rational $H$-characteristic numbers of $\partial_{o} \mathcal{W}(H)$ are zero.

Remark 3.2 Using $\partial_{o} \mathcal{W}(H)$, we see that when $[H] \in \beta_{n}^{-}$there is a closed, oriented orbifold with exactly two isolated singular points and the oriented vertex group for those points is $H$. If $H \in \beta_{n}^{+}$, we have a similar case, but the oriented vertex groups are $H$ and $\alpha H \alpha^{-1}$.

Suppose $N$ is an oriented $(n+1)$-dimensional orbifold with boundary $Q$ and $H$ and $\alpha H \alpha^{-1}, \alpha \in O(n)-\mathrm{SO}(n)$ are oriented vertex groups for the points $x, x^{\prime}$ in $Q$. Let $h$ and $h^{\prime}$ be orientation-preserving orbifold diffeomorphisms of $\dot{D}^{n} / H$, respectively $\dot{D}^{n} /\left(\alpha H \alpha^{-1}\right)$, to neighborhoods $U$, respectively $U^{\prime}$, of $x$, respectively $x^{\prime}$, which extend to diffeomorphisms of the closures.

Definition 3.3 Let $M=N \cup \mathcal{W}(H) / \sim$, where $y=\left(y_{1}, 0\right) \in \mathcal{W}(H)$ is identified with $h^{\prime}\left(y_{1}\right) \in \bar{U}^{\prime}$ and $y^{\prime}=\left(y_{1}^{\prime}, 1\right) \in \mathcal{W}(H)$ is identified with $h\left(y_{1}^{\prime}\right) \in \bar{U}$. Once the angle is straightened, $M$ is an oriented orbifold with boundary. The exchanging of vertex groups was to ensure the orientation. We say $M$ is obtained from $N$ by attaching an $(H, 1)$-handle.

More generally we have the following.

Definition 3.4 Suppose the finite linear group $G<\mathrm{SO}(n+1)$ decomposes as the direct sum $\phi_{1}(G) \oplus \phi_{2}(G)$, where the summands have degrees $k_{1}, k_{2}$. Suppose the locally oriented $n$-orbifold $Q$ has a closed neighborhood $U$ which is orbifold diffeomorphic to $\left(S^{k_{1}-1} \times D^{k_{2}}\right) / G$. In the manner analogous to that for manifolds as in Kosinski [8] or Ranicki [9], or tom Dieck and Hambleton [2] for equivariant surgery, we define a $\left(G, \phi_{1}(G)\right)$-surgery and when $Q$ is an orbifold boundary, attaching a $\left(G, \phi_{1}(G)\right)$-handle.

(i) The procedure of $\left(G, \phi_{1}(G)\right)$-surgery on $Q$ results in a new orbifold

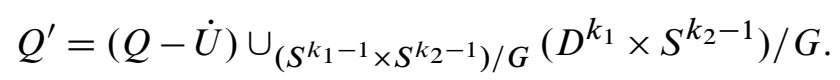

(ii) In the case where $Q=\partial_{o} M$ we form a new orbifold with boundary, $N$, by attaching a $\left(G, \phi_{1}(G)\right)$-handle to $M$ via

$$
N=M \cup_{\left(S^{k_{1}-1} \times D^{k_{2}-1}\right) / G}\left(D^{k_{1}} \times D^{k_{2}}\right) / G .
$$

Remark 3.5 If $Q$ is oriented, then $Q^{\prime}$ is oriented by giving the second summand negative the standard orientation and similarly for $M$ and $N$. 
Remark 3.6 We have $Q^{\prime} \sim_{o} Q$, where the cobordism is provided by attaching a handle to $Q \times I$ at $Q \times 1$. When $Q=\partial_{o} M, \partial_{o} N$ is obtained from $Q$ via a $\left(G, \phi_{1}(G)\right)-$ surgery.

Definition 3.7 When $\phi_{1}(G)$ is trivial, we refer to the above constructions as a $\left(\phi_{2}(G), k_{1}-1\right)$-surgery and attaching a $\left(\phi_{2}(G), k_{1}\right)$-handle. In this case, the surgery replaces a $\phi_{2}(G)$-singular set with singular sets with local groups from $\mathcal{G}\left(\phi_{2}(G)\right)$.

Proposition 3.8 When $G$ is a finite degree- $n$ subgroup of $\mathrm{SO}(n)$, some multiple of the sphere quotient orbifold $S^{n-1} / G$ bounds an oriented orbifold with set of local groups no more than those found in $S^{n-1} / G$. Thus one can construct an oriented orbifold $Q$ whose only vertex group is $G$, possibly with repetition.

Proof The quotient orbifold $S^{n-1} / G$ orientably bounds the disk quotient orbifold $D^{n} / G$, and so by [5, Lemma 2.1] some multiple $k$ of $S^{n-1} / G$ bounds some oriented orbifold $W$ with no increase in local groups. We then define $Q$ to be

$$
\left(k\left(D^{n} / G\right)\right) \cup_{k S^{n-1} / G}-W .
$$

Proposition 3.9 For $G$ a direct sum of complex type or symplectic type groups or $\left\langle-\mathrm{id}_{2 l}\right\rangle, S^{n-1} / G$ bounds orientably with no increase in local groups and $G$ can occur as the only vertex group, with no repetitions, in an oriented orbifold.

Proof In those cases $S^{1}$, considered as the standard diagonal subgroup of $U(j)<$ $\mathrm{SO}(2 j)$ for complex type groups and $\left\langle-\mathrm{id}_{2 l}\right\rangle$ and via $(\lambda, \bar{\lambda}) \in \mathrm{Sp}(1)<U(2)$ as a subgroup of $\mathrm{SO}(4 j)$ for symplectic type groups, and finally as an appropriate direct sum of these, commutes with the action of $G$. This affords us an action of $S^{1} \times G$ on $S^{n-1}$ with $S^{1} \cap G$ acting as the identity. Let $m=n / 2$, where $n$ is the degree of $G$. This action of $S^{1}$ on $S^{2 m-1}$ over $\mathbb{C} P^{m-1}$ descends to an $S^{1} /\left(S^{1} \cap G\right)$-fibering of $S^{2 m-1} / G$ over $\mathbb{C} P^{m-1} /\left(G /\left(S^{1} \cap G\right)\right)$. An orbifold $W$ is formed by replacing this fiber by $D^{2}$ to get that $S^{2 m-1} / G$ bounds with no increase in local groups. We get an orbifold with one vertex group, which is labelled by $G$, by the construction in the proof of Proposition 3.8 with $k=1$.

When $G$ is as in Proposition 3.9, $d[G]=0$, since no subgroup is of codegree 1. In general, if one tries to construct a closed locally oriented $n$-orbifold with an isolated singular point labelled by $G$, one sees that extending out from the point in $D^{n} / G$ one has the singular line segments labelled by the vertex groups of $G$. These segments will have to match up, since there is only one terminal point for them. Hence we must have $d[G]=0$. This generalizes to multiple isolated singular points, but now the labelled singular edges may connect these points. This motivates our following definition to relate orbifolds to $d$. 
Definition 3.10 Define a map $\psi$ from the set of diffeomorphism classes of closed locally oriented orbifolds to $\beta$ as follows. For $Q$ a compact locally oriented $n-$ dimensional orbifold let the isolated singular points in $Q$ be denoted by $x_{1}, x_{2}, \ldots, x_{k}$ and their associated vertex groups by $G_{1}, G_{2}, \ldots, G_{k}$. The group at $x_{i}$ is well defined up to conjugacy in $\mathrm{Gl}(n, R)$, or since a Riemannian metric can be provided, lies in $\mathrm{SO}(n)$, and since it is well defined up to conjugacy in $\operatorname{Gl}(n, R)$ it is likewise well defined in $O(n)$. Define $\psi(Q) \in \beta_{n}$ as $\sum_{1}^{k}\left[G_{i}\right]$. Then $\psi$ is a ring homomorphism with disjoint union and Cartesian product the operations on the set of orbifolds.

Remark 3.11 If $(G) \in \mathcal{S O}_{n, f}, \psi\left(S^{n-1} / G\right)=d[G]$. We concentrated on just $d[G]$ in previous sections to allow the algebra and geometry of representations to aid us in calculating $\operatorname{ker} d$ and $\mathcal{H}_{n}$.

Theorem 3.12 (i) If $Q$ is a closed locally oriented $n$-dimensional orbifold, then $d(\psi(Q))=0$. Hence $d \sum_{i=1}^{k}\left[G_{i}\right]$ provides a first obstruction to whether a set of degree- $n$ groups $G_{1}, G_{2}, \ldots, G_{k} \in \mathrm{SO}(n)$ can be the set of vertex groups for a locally oriented closed $n$-dimensional orbifold.

(ii) If $Q=\partial_{o} W$, then $\psi(Q) \in \operatorname{im} d_{n+1}$, and so $\psi$ induces a map $\bar{\psi}: \Omega_{n, \text { orb }} \rightarrow \mathcal{H}_{n}$.

(iii) Suppose $Q$ is oriented. If $\psi(Q) \in \operatorname{im} d_{n+1}$ and all its rational $H$-numbers are zero for any $H$ a vertex group, then $Q \sim_{o} Q^{\prime}$, where $Q^{\prime}$ has local groups of degree less than $n$.

(iv) Suppose $G$ has degree $n+1$. If $Q^{\prime}$ is obtained from the orbifold $Q$ by a $\left(G, \phi_{1}(G)\right)$-surgery, $\psi\left(Q^{\prime}\right)=\psi(Q)+d[G]$.

Proof (i) Let $V_{i}, 1 \leq i \leq k$, be disjoint closed neighborhoods of the $x_{i}$ such that there are orbifold diffeomorphisms $f_{i}: D^{n} / G_{i} \rightarrow V_{i}$ for $i=1, \ldots, k$. Then $Q-\bigcup_{1}^{k} \dot{V}_{i}$ has no vertex groups. If a point $y$ in $S^{n-1} / G_{i}$ has a degree- $(n-1)$ local group $H$, then the singular edge with local group $H$ in $Q-\bigcup_{1}^{k} \dot{V}_{i}$ emanating from $f_{i}(y)$ must end at a different point (labelled $H$ ) in some $f_{j}\left(S^{n-1} / G_{j}\right) \subset Q-\bigcup_{1}^{k} \dot{V}_{i}$.

(ii) Let $H_{1}, H_{2}, \ldots, H_{l}$ be the (degree $n+1$ ) vertex groups of the isolated singular points $x_{1}, \ldots, x_{l}$ interior to $W$, with possible repetition in vertex group. Suppose $V_{1}, \ldots, V_{l}$ are disjoint closed neighborhoods of these points and $f_{i}: D^{n+1} / H_{i} \rightarrow V_{i}$ for $i=1, \ldots, l$ are oriented orbifold diffeomorphisms. Then either a vertex point with (degree $n$ ) group, say $K$, on the $\bigcup_{1}^{l} f_{i}\left(S^{n} / H_{i}\right)$ is linked by an edge labelled $K$ internal to $W$ to another vertex point on it (hence even parity and contributing nothing to $d \sum_{1}^{l}\left[H_{i}\right]$ ), or the edge terminates in $Q$ and $[K]=\left[G_{i}\right]$ for some $i$. Also every vertex point on $Q$ is the terminus of a labelled edge in $W$. Therefore $\sum_{1}^{k}\left[G_{i}\right]=d \sum_{1}^{l}\left[H_{i}\right]$. 
(iii) Suppose that $\psi(Q)=d \sum_{i=1}^{l}\left[K_{i}\right]$, where this is a minimal sum. Let $W_{1}=$ $(Q \times I) \amalg \bigsqcup_{i=1}^{l} D^{n+1} / K_{i}$. We will attach handles to $W_{1}$ as follows. First we can assume that $A=\left\{G_{1}, \ldots, G_{j}\right\}$ for some $j \leq k$ is a nonrepeating list of the vertex groups which occur with odd parity in $Q$.

Since $\psi(Q)=d \sum_{i=1}^{l}\left[K_{i}\right]$ the groups in $A$ must be those which occur with odd parity in $\bigsqcup_{i=1}^{l} S^{n} / K_{i}$. Because of that, they all come from $\beta_{n}^{-}$. For each $1 \leq i \leq j$ we attach a $\left(G_{i}, 1\right)$-handle connecting $\left(x_{i}, 1\right)$ to a point in $\coprod_{i=1}^{l} S^{n} / K_{i}$ with vertex group $G_{i}$.

The remaining vertex groups for the remaining isolated singular points in $Q$ must occur in pairs. We can then attach $\left(G_{S}, 1\right)$-handles for these pairs of points in $Q \times 1$. When $\left[G_{s}\right] \in \beta_{n}^{+}$, we assume $G_{s}$ is the oriented vertex group of $x_{s}$. The fact that the $G_{S}$-characteristic numbers of $Q$ are zero implies that another point with vertex group $G_{S}$ has an opposite oriented vertex group. This is how we pair our points in attaching handles to assure the resulting orbifold is oriented.

We now look at the remaining vertex groups for $\bigsqcup_{i=1}^{l} S^{n} / K_{i}$. As in the previous paragraph these must occur in pairs. Since $S^{n} / K_{i}=\partial_{o} D^{n+1} / K_{i}$, the $H$-characteristic numbers of $S^{n} / K_{i}$ are zero for $H$ a vertex group for some $K_{i}$. Hence we can proceed as in the previous paragraph and attach an $(H, 1)$-handle to $D^{n+1} / K_{i}$. We do this for all such $H$. The resulting orbifold with boundary provides the cobordism between $Q$ and $Q^{\prime}$. We have that $Q^{\prime}$ is $Q \amalg \bigsqcup_{i=1}^{l} S^{n} / K_{i}$ surgered at all its vertex points.

(iv) We have that $\left.Q^{\prime}=\left(Q-\left(S^{k_{1}-1} \times D^{k_{2}}\right) / G\right) \cup \sim_{i}\left(D^{k_{1}} \times S^{k_{2}-1}\right) / G\right)$, so $Q^{\prime}$ loses the isolated singular points contributing vertex groups in the first summand $S_{1}$ of Proposition 2.1, and it gains those associated with the second summand $S_{2}$. Hence $\psi\left(Q^{\prime}\right)=\psi(Q)-S_{1}+S_{2}$. But in $Z_{2}$ coefficients, this is $\psi(Q)+S_{1}+S_{2}=$ $\psi(Q)+d[G]$.

Theorem 3.13 Suppose $n \leq 4$. If $G_{1}, G_{2}, \ldots, G_{k}$, are finite degree- $n$ subgroups of $\mathrm{SO}(n)$ and $\sum_{i=1}^{k} d\left[G_{i}\right]=0$, there is a closed, oriented $n$-orbifold $Q$ whose vertex groups are precisely those groups. Hence $\psi$ maps onto $\operatorname{ker} d_{n}$.

Proof For dimension two we have ker $d_{2}=\beta_{2}=\bigoplus_{k \geq 2}\left[\mathcal{C}_{k}\right]$. Thus for any linear combination $L=\sum_{s=1}^{k}\left[\mathcal{C}_{j_{s}}\right], d L=0$. The orbifold boundary of $Q_{1}=\coprod_{s=1}^{k} D^{2} / \mathcal{C}_{j_{s}}$ is diffeomorphic to $k$ copies of $S^{1}$. One choice for $Q$ is $Q=\left(S^{2}-\coprod_{s=1}^{k} U_{s}\right) \cup_{f} Q_{1}$, where the $U_{s}$ are disjoint disc neighborhoods whose boundary $S$ is diffeomorphic to $\coprod_{s=1}^{k} S^{1}$ and $f: S \rightarrow \partial_{o} Q_{1}$ is an orientation-reversing diffeomorphism. 
We now consider the case where $n$ is three or four. Let $N_{1}=\coprod_{i=1}^{k} D^{n} / G_{i}$ and $Q_{1}=\coprod_{i=1}^{k} S^{n-1} / G_{i}$, the orbifold boundary of $N_{1}$. Suppose $H$ is a vertex group for $Q_{1}$. Since $\sum_{i=1}^{k} d\left[G_{i}\right]=0$, and when $n$ is three or four, $\beta_{n-1}=\beta_{n-1}^{-}, H$ occurs as an oriented vertex group for an even number of points in $Q_{1}$. We then attach $(H, 1)$-handles to $N_{1}$ at all these points and repeat this for each such $H$ to form a new oriented orbifold with boundary which we will denote by $N_{2}$. By construction, $\partial_{o} N_{2}$ has no vertex groups.

For $n=3$ this means that $\partial_{o} N_{2}$ is an oriented 2-dimensional manifold and bounds an oriented manifold, say $W$. We let $Q=N_{2} \cup-W / \sim$, where $N_{2}$ and $-W$ are glued together by the identity map along their boundaries.

For $n=4, \partial_{o} N_{2}$ is an oriented 3-orbifold with no vertex groups. Hence it is either an oriented 3-manifold or its singular set consists of circles. In the former case we can proceed as when $n=3$, since from Rohlin [10] we have that any oriented 3-manifold bounds an oriented 4 -dimensional manifold.

In the latter case, its singular set is $\bigsqcup_{s=1}^{m} V_{s}$, where each $V_{s}$ is diffeomorphic to $S^{1}$ and has a closed tubular neighborhood $\mathcal{N}\left(V_{S}\right)$ which is oriented orbifold diffeomorphic to the total space of a $\left(D^{2} / \mathcal{C}_{l_{s}}, N_{O(2)}\left(\mathcal{C}_{l_{s}}\right) / \mathcal{C}_{l_{s}}\right)$-orbibundle $\zeta_{s}$ over $S^{1}$. Such a bundle is classified by a map $f: S^{1} \rightarrow B N_{O(2)}\left(\mathcal{C}_{l_{s}}\right) / \mathcal{C}_{l_{s}}$. We have $N_{O(2)}\left(\mathcal{C}_{l_{s}}\right)=O(2)$ and $O(2) / \mathcal{C}_{l_{s}} \cong O(2)$ and, since the total space is oriented, as is $S^{1}$, we can reduce the structure group to $\mathrm{SO}(2) \cong S^{1}$. Now $\pi_{1}\left(B S^{1}\right)=0$, so $\zeta_{S}$ is trivial, and hence has total space diffeomorphic to $S^{1} \times D^{2} / \mathcal{C}_{l_{s}}$. This allows us to create $N_{3}$ by attaching $\left(\mathcal{C}_{l_{s}}, 2\right)$-handles along the $\mathcal{N}\left(V_{s}\right)$. Since $\partial_{o} N_{3}$ is an oriented 3-manifold, it bounds a manifold $B$. We let $Q=\left(N_{3} \cup-B\right) / \sim$, where the two pieces are sewn together along $\partial_{o} N_{3}$.

\section{References}

[1] A Adem, J Leida, Y Ruan, Orbifolds and stringy topology, Cambridge Tracts in Math. 171, Cambridge Univ. Press (2007) MR2359514

[2] T tom Dieck, I Hambleton, Surgery theory and geometry of representations, DMV Seminar 11, Birkhäuser, Basel (1988) MR946473

[3] J D Dixon, Problems in group theory, Dover, Mineola, NY (1967) MR0218428

[4] K S Druschel, Oriented orbifold cobordism, Pacific J. Math. 164 (1994) 299-319 MR1272653

[5] K S Druschel, The cobordism of oriented three-dimensional orbifolds, Pacific J. Math. 193 (2000) 45-55 MR1748181 
[6] P Du Val, Homographies, quaternions and rotations, Clarendon Press, Oxford (1964) MR0169108

[7] I M Isaacs, Finite group theory, Graduate Studies in Math. 92, Amer. Math. Soc. (2008) MR2426855

[8] A A Kosinski, Differential manifolds, Pure and Applied Math. 138, Academic Press, Boston (1993) MR1190010

[9] A Ranicki, Algebraic and geometric surgery, Clarendon Press, Oxford (2002) MR2061749

[10] V A Rohlin, A three-dimensional manifold is the boundary of a four-dimensional one, Doklady Akad. Nauk SSSR 81 (1951) 355-357 MR0048808

Department of Mathematics and Computer Science, Saint Louis University 221 N. Grand Blvd., Saint Louis, MO, USA

druschelks@slu.edu

Received: 1 November 2013 Revised: 23 May 2014 